\title{
Neuroleptic Treatment of HIV-Associated Psychosis
}

Daniel D. Sewell, M.D., Dilip V. Jeste, M.D., Lou Ann McAdams, Ph.D., Anne Bailey, M.S., M. Jackuelyn Harris, M.D., J. Hampton Atkinson, M.D., James L. Chandler, M.D., J. Allen McCutchan, M.D., Igor Grant, M.D., and the HNRC Group*

The aim of this rater-blinded randomized study was to evaluate the efficacy and side effects of haloperidol and thioridazine in the treatment of new-onset psychosis in HIV-positive individuals. Participants were 13 men who had no history of psychosis prior to infection with HIV, and whose psychosis was not attributable to delirium or to non-HIV-related organic factors. Participants were evaluated at baseline after at least one month without neuroleptic treatment and then weekly for six weeks of the experimental treatment using several rating scales. The mean daily dose in chlorpromazine equivalents was
$124 \mathrm{mg}$. Both neuroleptics produced modest but significant reduction in overall level of psychosis and in positive symptoms, but not in negative symptoms. All the haloperidol-treated patients developed extrapyramidal side effects and required treatment with anticholinergic medication, whereas three of the five thioridazine-treated patients had noticeable side effects. We make recommendations for the treatment of HIV-associated psychosis with neuroleptics.

[Neuropsychopharmacology 10:223-229, 1994]
KEY WORDS: Tranquilizing agents, major; Organic mental disorders; Haloperidol; Thioridazine; Schizophrenia; AIDS.

From the University of California (DDS, DVJ, LAM, AB, MJH, JHA, JAM, IG), San Diego, San Diego VA MedicalCenter(DDS, DV], MJH, JHA, IG), Naval Hospital San Diego (JLC) in San Diego, California. Address all correspondence to: Daniel D. Sewell, M.D., Psychiatry Service (116A), San Diego VA Medical Center, 3350 La Jolla Village Drive, San Diego, California 92161

* The San Diego HIV Neurobehavioral Research Center (HNRC) Group is affiliated with the University of California, San Diego, the Naval Hospital, San Diego, and the San Diego VA Medical Center, and includes: Igor Grant, M.D., Director; J. Hampton Atkinson, M.D., Co-Director; Robert A. Velin, Ph.D., Center Manager; Edward C. Oldfield III, M.D., James L. Chandler, M.D., Mark R. Wallace, M.D., and Joseph Malone, M.D., Co-Investigators Naval Hospital San Diego; J. Allen McCutchan, M.D., P.I. Medical Core; Stephen A. Spector, M.D., P.I. Virology Core; Leon Thal, M.D., P.I. Neurology Core; Robert K. Heaton, Ph.D., P.I. Neuropsychology Core; John Hesselink, M.D. and Terry Jernigan, Ph.D., Co-P.I.s Imaging Core; J. Hampton Atkinson, M.D., P.I. Psychiatry Core, Clayton A. Wiley, M.D., Ph.D., P.I. Neuropathology Core; Richard Olshen, Ph.D. and Ian Abramson, Ph.D., Co-P.I.s Biostatistics Core; Nelson Butters, Ph.D., P.I. Memory Project; Renee Dupont, M.D., P.I. SPECT Project; Thomas
The psychiatric sequelae of infection with HIV include depression, anxiety, delirium, dementia, and psychosis (Fernandez and Levy 1991). How often patients with no past history of psychosis develop psychotic symptoms subsequent to infection with HIV is not known (Harris et al. 1991; Sewell et al. 1994). The available information suggests that new-onset psychosis in individuals infected with HIV, although not common, has serious potential consequences (Miller and Riccio 1990; Boccellari and Dilley 1992).

Although a causal relationship between the presence of HIV in the central nervous system and the development of psychosis has not been established with certainty, there is a growing body of scientific information that supports this notion (Masdeu et al. 1991; Mas-

Patterson, Ph.D., P.I. Life Events Project; Sidney Zisook, M.D., P.I. Mood Project; Dilip Jeste, M.D., P.I. Psychosis Project; Hans Sieburg, Ph.D., P.I. Dynamical Systems Project; and James D. Weinrich, Ph.D., P.I. Sexology Project.

Received August 16, 1993; revised January 5, 1994; accepted January 31, 1994. 
liah et al. 1992). These findings provide a possible explanation for the development of psychotic symptoms as well as for the reported sensitivity to side effects of psychotropic medications in general (Perry and Jacobsen 1986; Gilmer and Busch 1991) and neuroleptics in particular (Breitbart 1988; Goodkin 1988; Harris et al. 1991; Hriso et al. 1991).

We found no systematic, prospective studies of the treatment of new-onset psychosis in patients infected with HIV. Information in the literature is currently limited to retrospective chart reviews and case reports, and there is no consensus regarding whether highpotency or low-potency neuroleptics are preferable. HIV-infected patients have been reported to be much more sensitive to extrapyramidal side effects (EPS) associated with neuroleptic medication, even when the age and gender of the patients are considered (Hriso et al. 1991). On the other hand, anticholinergic side effects must be minimized in order to avoid exacerbating preexisting memory deficits and to avoid increasing the risk of candidiasis secondary to dry mucous membranes. Low-potency (more anticholinergic) neuroleptics may also cause confusion when used to treat HIV-infected patients (Harris et al. 1991). Although high-potency neuroleptics have the disadvantage of being much more likely to cause EPS, they provide more antidopaminergic blockage per $\mathrm{mg}$ and are less anticholinergic.

The purpose of our investigation was to study the efficacy and side effects of two commonly used neuroleptics of widely different potencies in the treatment of HIV-associated psychosis. We specifically hypothesized that HIV-infected patients would (1) obtain greater reduction in psychotic symptoms with haloperidol than with thioridazine and (2) experience fewer overall side effects with haloperidol than with thioridazine.

\section{MATERIALS AND METHODS}

\section{Subjects}

Patients who were included in the present investigation came from a comprehensive neuropsychiatric study of new-onset psychosis in 33 patients infected with HIV (Sewell et al. 1994). That study was, in turn, a part of the San Diego HIV Neurobehavioral Research Center (HNRC), a prospective investigation designed to examine the neuropsychiatric consequences of infection with HIV. Patients in the comprehensive neuropsychiatric study of new-onset psychosis complete an extensive assessment that has been previously described (Sewell et al. 1994).

Patients in the present protocol met the following criteria: (1) age between 18 and 50 years; (2) infection with HIV as determined by ELISA and confirmed by Western Blot; (3) presence of delusions or prominent hallucinations; (4) no history of psychosis prior to infection with HIV; (5) no illicit drug use during three months prior to the onset of psychotic symptoms; (6) absence of delirium as defined by DSM-III-R (American Psychiatric Association 1987); (7) neuroleptic-free for at least a month prior to entry; and (8) willing to provide written consent for participation. Seventeen of the 33 patients met selection criteria for the study. The reasons (with $\mathrm{N}$ ) for the exclusion of patients were: already taking neuroleptic at the time of enrollment and refusing drug-withdrawal (11); refusal to take neuroleptic (4); and absence of psychosis at the time of enrollment (1). Thirteen patients completed the study (i.e., completed at least three sets of weekly ratings) and four dropped out. Three of the dropouts were administrative, two because urine toxicology studies done during the study revealed amphetamine use and one because severe medical illness prevented the patient from completing the clinical ratings. In regards to the two patients who were excluded due to amphetamine use, our documentation of amphetamine use led us to conclude that all of the data provided by these patients could have been contaminated by amphetamine use (i.e., perhaps they were using amphetamine even before we discovered that they were). Regarding the patient who became too ill to complete the evaluations, only one set of post baseline data was available. The fourth patient did not return after baseline evaluation. The mean (with standard deviation [SD]) age of the 13 male patients who completed the study was $36(8)$ years and the mean years of education completed (with SD) was 13 (2) years. The majority of these patients were caucasian $(n=11)$ and were in CDC stage C (AIDS) $(n=7)$ (Center for Disease Control and Prevention 1992), and their mean absolute serum T4 cell count (with SD) was 308 (258) cells/ $\mu \mathrm{l}(n=12)$. Eleven of the 13 subjects underwent magnetic resonance imaging (MRI) of the brain. None of these images revealed opportunistic infections or neoplasms. Ten of the 13 patients were taking zidovudine (AZT) at the time of enrollment.

\section{Study Design}

Patients entered the study as inpatients or as outpatients. At the time of the initial contact, all the patients were given the option of receiving psychiatric care from one of the authors (D.D.S.) and all but two patients accepted this option. Using a 1:1 randomization strategy, patients were assigned to treatment with haloperidol or thioridazine. The mean (with standard deviation) starting dose for patients treated with haloperidol was 48 (41) $\mathrm{mg}$, and the mean starting dose for patients treated with thioridazine was $86(46) \mathrm{mg}$. Decisions regarding optimal neuroleptic dose were made by D.D.S. Optimal dose was established clinically by balancing maximum possible reduction in psychotic symptoms 
against minimum possible side effects. In general, each patient's dose was gradually increased over the sixweek study period. Patients were not treated with an anti-EPS medication on a prophylactic basis. Patients who developed EPS received benztropine mesylate $(n=8)$ or trihexyphenidyl hydrochloride $(n=1)$. After the initial six-week period, patients were seen as often as necessary based on their clinical status.

At entry and for the first six weeks of treatment, a rater who was blinded to neuroleptic assignment completed weekly evaluations of each of the patients for efficacy and side effects using the 26-item version of the Brief Psychiatric Rating Scale (BPRS) (Overall 1988), Scale for the Assessment of Positive Symptoms (SAPS) (Andreasen and Olsen 1982), Scale for the Assessment of Negative Symptoms (SANS) (Andreasen and Olsen 1982), Simpson-Angus' EPS rating scale (Simpson and Angus 1970), and Abnormal Involuntary Movement Scale or AIMS (National Institute of Mental Health 1976). Ratings were completed by one of three individuals who were from a pool of raters who achieved good interrater reliability as measured by intraclass correlation coefficients ranging from .77 to .86 on the rating scales used. The blind was maintained by instructing patients to refrain from discussing their medications with the raters and by excluding the raters from access to the patients' charts. Eight of the 11 patients randomized to treatment with haloperidol completed the study. The mean maximum daily dose of haloperidol (with SD) for these eight patients was 2.9 (1.4) $\mathrm{mg}$ or 120 (61) in mg chlorpromazine equivalents (Jeste and Wyatt 1982). Five of the six patients randomized to treatment with thioridazine completed the protocol. The mean daily dose of thioridazine (with standard deviation) for these six patients was 145 (76) $\mathrm{mg}$ or 131 (68) in $\mathrm{mg}$ chlorpromazine equivalents. There was no significant difference in dose in chlorpromazine equivalents between the two groups.

\section{Statistical Analysis}

We compared the 13 patients who completed the treatment study with the other 20 psychotic patients who did not participate on demographic, clinical rating scale, magnetic resonance imaging, cerebral spinal fluid, and neuropsychological variables to determine if individuals who met criteria for inclusion for and completed the treatment study differed in other ways from those who did not meet inclusion criteria or who did not complete the study. For continuous variables we used two-tailed $t$-tests unless the assumptions for parametric statistics were not met, in which case we used two-tailed MannWhitney U-tests. For categorical variables (race and CDC stage) we used chi-square analysis.

The treatment study could be conceptualized as a split-plot design with a drug grouping factor and a repeated measures time factor (baseline and weeks 1 to 6) (Kirk 1982). Ordinarily this would call for the use of repeated-measures analysis of variance procedure. Missing data, however, led us to select the Newton Raphson (NR) maximum likelihood approach to estimate parameters and to test the time effect, the drug group effect, and drug group by time interaction effect (Schluchter 1988; Dixon et al. 1990).

In order to justify the adoption of this approach, we assumed that any missing data were missing at random. In order to run the BMDP statistical software (Dixon et al. 1990) version of the NR maximum likelihood approach $(5 \mathrm{~V})$, we also had to assume a particular structure for the pooled variance-covariance matrix (that is, pooled across the two groups). For each of the rating scales with the exception of the AIMS total score, we assumed equal variances and equal covariances in the pooled variance-covariance matrix, and for the AIMS total score we assumed a banded structure for the variance-covariance matrix (equal variances in the main diagonal of the matrix and equal covariances within paired symmetrical bands above and below the main diagonal of the matrix).

In addition to testing the main effects of drug and time, and the drug by time interaction, we tested the first three orthogonal trend components of the time effect: linear, quadratic, and cubic, and their interactions with the drug factor. Finally, we correlated the change in BPRS total score with baseline full-scale IQ using Spearman's correlation (rho).

\section{RESULTS}

The group of patients who completed the treatment study $(n=13)$ did not differ from the group of patients who did not participate in or complete the study $(n=$ 20 ) on age, race, education, CDC stage, any of the rating scale total scores, neuropsychological global scores, absolute T4 count, serum P24 level, or MRI global ratings.

Treatment with neuroleptics resulted in a signifcant reduction in psychopathology as represented in the BPRS total score and in the BPRS disorganization subscale score which is composed of four items. The mean BPRS total score (68 at baseline and 54 at week six) after rescaling to reflect a minimum possible score of 24 decreased $32 \%$ by the end of the study, while the BPRS disorganization subscale score (mean 9.5 at baseline) decreased by $62 \%$. Figure 1 depicts the estimated values for the BPRS disorganization subscale score over the first six weeks of treatment. Nonsignificant improvements were observed in BPRS hostility subscale. In regards to the SAPS total score, whereas the overall time factor was not significant, the linear time component was significant $(p=.022)$. We did not observe im- 


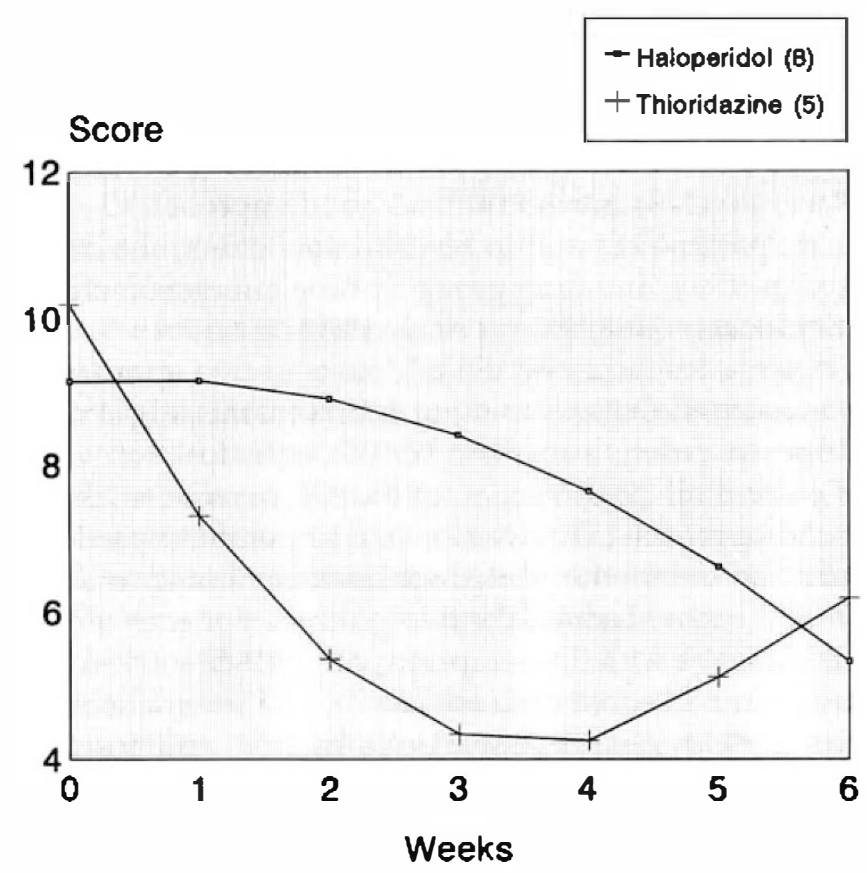

Figure 1. This figure depicts the predicted curves from a repeated measures model for BPRS disorganization subscale score over a six-week treatment period for eight psychotic HIVinfected patients randomly assigned to treatment with haloperidol and five psychotic HIV-infected patients randomly assigned to treatment with thioridazine. The overall estimated mean scores averaged across time for the two groups were not significantly different. The time affect across both groups was significant $(p=.001)$. In addition, the group $\times$ time interaction was significant $(p=.003)$. The group $\times$ linear time component did not contribute to this difference; however, the group $\times$ quadratic time component did $(p=.0005)$.

provements in the BPRS depression subscale or the SANS total. Figure 2 presents the mean SAPS and SANS total scores over the first six weeks of treatment. AIMS total score increased nonsignificantly over time.

Haloperidol and thioridazine were similar in that both resulted in a reduction in psychopathology. The reduction in BPRS total was linear in both groups, but the reduction in BPRS disorganization was curvilinear, with the thioridazine-induced reduction occurring earlier and haloperidol-induced reduction occurring later in the six-week period. There was significantly greater improvement in BPRS hostility subscale score (and also a nonsignificantly greater improvement in SAPS total) in the thioridazine group. There was no significant correlation between full-scale IQ at baseline and change in BPRS total score.

In terms of side effects, none of the patients experienced symptomatic orthostasis, although pulse and blood pressure were not measured routinely. All of the eight patients on haloperidol developed EPS and required treatment with benztropine. One patient treated with haloperidol developed TD and another patient ex-

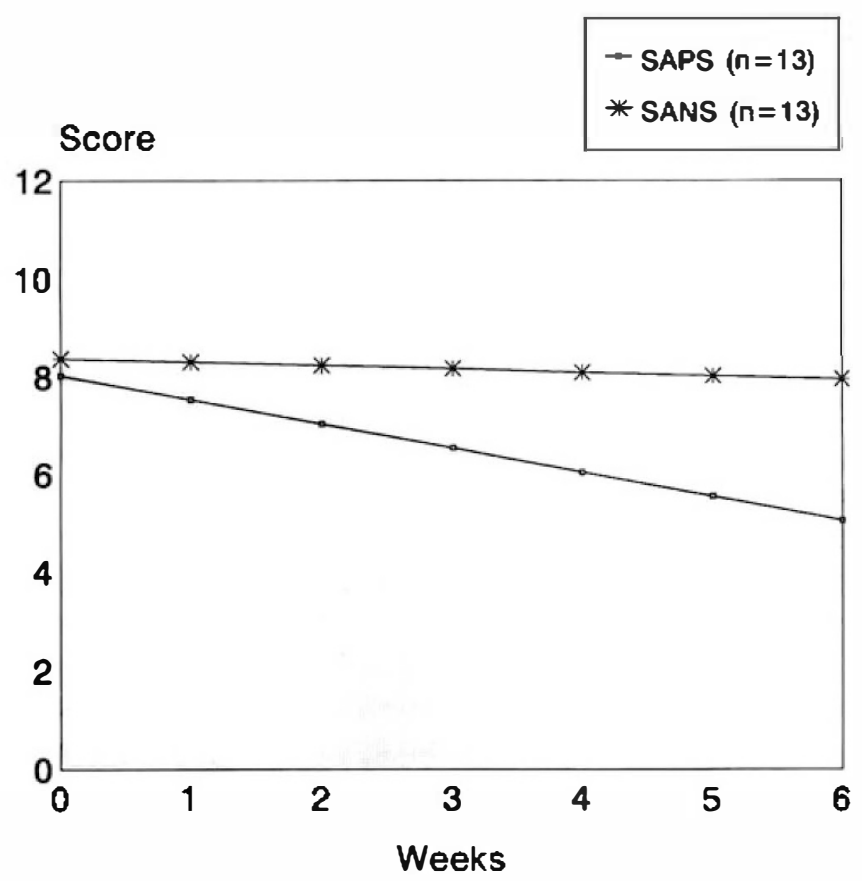

Figure 2. This figure depicts the predicted line calculated from a repeated measures model for SAPS and SANS rating scale scores over a six-week treatment period for 13 psychotic HIV-infected patients. The linear component of time trend was significant for the SAPS ( $p=.022)$ but not for the SANS.

perienced sedation. Three of the five patients treated with thioridazine had problematic side effects. One patient on thioridazine developed EPS and was treated with trihexyphenidyl. A second patient treated with thioridazine experienced weight gain, retrograde ejaculation, and dry mouth and developed TD. (The patients who developed TD with haloperidol and thioridazine met the published criteria for TD [Schooler and Kane 1982; Jeste and Wyatt 1982] except that the duration of neuroleptic treatment was less than three months.) A third patient treated with thioridazine had noticeable sedation.

\section{DISCUSSION}

Our study found a modest but significant improvement in the overall level of HIV-associated psychosis, especially positive symptoms, but not in negative symptoms, with six weeks of treatment with either haloperidol or thioridazine. The drug doses were relatively low, with a high incidence of side effects, mostly EPS with haloperidol.

Our study has several limitations. The number of patients studied was small and despite our best efforts, we had some missing data. As a result, we selected a statistical method that is applicable even when some 
data are missing (Schluchter 1988). This statistical method, however, requires caution when used with small samples. The use of a completely randomized procedure rather than a stratified randomization procedure resulted in an imbalance in the size of the two groups which, in turn, resulted in a reduction of power. The patients were not "blind," and there was no placebo period. Clinical and practical issues required us to inform each patient about which drug he was taking. For example, if a patient developed an acute medical problem (which was not rare), then the patient needed to provide his physicians with the names and doses of all of the medications that he was taking in order to receive an optimal evaluation. It was prudent for us to underestimate the ability of our sick patients to cope with a system of allowing the "blind" to be broken in the face of a medical emergency. We had originally planned to have a placebo treatment period; however, the number and intensity of the patients' psychotic symptoms at the time of presentation led us to eliminate the placebo period from our protocol for ethical and humanitarian reasons.

On the other hand, to our knowledge this is the largest prospective study of the use of neuroleptic medication for the treatment of new-onset psychosis in patients infected with HIV. In addition, our protocol included well-defined subjects and rater-blinded assessments using well-established rating scales administered by raters who achieved a good level of interrater reliability.

Although our sample size was small, we should point out that HIV-associated psychosis is not a common condition. Furthermore, there are difficulties in studying these patients. In general, our patients faced more than the usual number of impediments to participation in psychiatric clinical research (Sewell et al. 1994). The majority of our patients had AIDS, and many had medical symptoms such as weakness and fatigue. Some of our subjects had difficulty participating because of a lack of family support and limited social and financial resources.

The main reason that we chose to study haloperidol and thioridazine was to determine whether high-potency or low-potency medications were better for the treatment of psychosis in patients infected with HIV. Furthermore, these two neuroleptics belong to different chemical classes and have different side effect profiles. We chose a six-week study period based on the results of the literature review by Davis et al. (1989) who found that in schizophrenic patients, most of the therapeutic gain occurred within the first six weeks of therapy with antipsychotic medication. We required that patients be neuroleptic-free for at least one month prior to entry in order to allow any nondepot neuroleptic that they might have already received to wash out as much as possible. (None of our patients was on de- pot neuroleptics.) We adopted a flexible dose schedule because of the paucity of information in the literature regarding optimal dose for the treatment of psychotic symptoms in HIV-infected patients. We chose not to use anti-EPS agents prophylactically, because we wanted to avoid the risks associated with the use of anticholinergic medications, and because we hoped that our study would allow us to estimate the frequency of EPS associated with the neuroleptics included in our study. The rating scales included in our design were selected because they have been commonly used, are readily available, and are associated with a large amount of published data regarding their use in other psychiatric cohorts.

In general, the nature and time course of neuroleptic response in our patients appeared to resemble those described by Davis et al. (1989) in acute schizophrenic patients. With six weeks of treatment with neuroleptic, the patients in our study experienced improvement in BPRS total score. Importantly, our patients required (or tolerated) a much smaller daily dose of antipsychotic medication than would be used to treat a patient with psychosis in other contexts. Currently, the optimal daily dose level in chlorpromazine equivalents for most "acute patients" with psychosis is 400 to $700 \mathrm{mg}$ (Davis et al. 1989). The mean maximum dose in chlorpromazine equivalents for our patients was $124 \mathrm{mg}$. Thislowerdose is similar to the lower doses used to treat older schizophrenic patients (Jeste et al. 1993) and patients with dementia (Wragg and Jeste 1988). Our findings are consistent with the recommendations of Ostrow et al. (1988) and Gilmer and Busch (1991) that psychotic symptoms in HIV-positive patients be treated with low dosages of neuroleptics. Why HIV-positive patients require, or more likely, tolerate lower doses of antipsychotic is unclear, but this could be related to pharmacokinetic changes due to chronic diseases (e.g., decreased hepatic clearance).

In a prospective study of 38 patients with AIDS who had organic mental disorders (all with delirium, although some had delirium and dementia or delirium and some other organic mental disorder) and were treated in an open trial with intravenous haloperidol and lorazepam, Fernandez et al. (1989) found that nearly half of the subjects experienced EPS and that patients with delirium along with some other organic mental disorder appeared to experience EPS more frequently than those with only delirium. Holmes (1989) described the successful treatment of an AIDS patient with monosymptomatic hypochondriacal psychosis with pimozide $(2 \mathrm{mg} /$ day). This patient experienced cogwheel rigidity and akathisia that responded to lowdose diazepam. The increased sensitivity of HIV-positive patients to neuroleptic-induced EPS suggests that damage to basal ganglionic structures could also be responsible for altered pharmacodynamics. 
Our preliminary results do not clearly demonstrate whether low or high potency neuroleptics are better for HIV-positive patients with psychosis. Our results do provide the basis for recommending relatively low doses of neuroleptic coupled with close monitoring for side effects, especially EPS and TD.

Additional information regarding pharmacokinetic and pharmacodynamic changes in patients with HIVassociated psychosis is needed. In our study, the mean improvement in total BPRS score over six weeks of treatment was only $32 \%$, although the mean BPRS disorganization subscale score decreased by $62 \%$. It is possible that a longer study period could reveal that HIV-infected patients with psychosis experience a greater magnitude of improvement and continue to improve steadily. It remains unclear how best to treat the negative symptoms that do not respond to "typical" neuroleptics. Clinical trials of clozapine or methylphenidate may prove useful.

\section{ACKNOWLEDGMENTS}

The principal support for this study was the HNRC. The principal support for the HNRC is provided by NIMH Center grant 5 P50 MH45294 (HIV Neurobehavioral Research Center). Additional support is provided by 5 R01 MH43298 (Neuropsychiatric Sequelae of HTLV-III Infections), 5 R01 MH45688 (Object-Oriented Simulation of HIV- and CNS/HIV Infection), 1 R01 MH 46255 (Psychosocial Moderators of Disease Progression in AIDS), 1 R01 NS27810 (Role of Immune Responsiveness in HIV Encephalopathy), and the Henry M. Jackson Foundation (Psychiatric Natural History Study: Factors Related to Human Immunodeficiency Virus Transmission and Morbidity). This work was also supported, in part, by NIMH grants MH43693-05 (Late-onset Schizophrenia: A Neuropsychiatric Study), MH45131-04 (Risk Factor for Tardive Dyskinesia in Older Patients) and MH49671-01 (CRC on Psychotics and Antipsychotics in Late Life) and by the Department of Veteran Affairs. The views expressed in this article are those of the authors and do not reflect the official policy or position of the Department of the Navy, Department of Defense, nor the United States Government. The authors acknowledge the valuable assistance of Brenda Clemons and Alice Schalz, R.N., in the preparation of the manuscript.

\section{REFERENCES}

American Psychiatric Association, (1987): Diagnostic and Statistical Manual of Mental Disorders, Third EditionRevised, Washington: American Psychiatric Association

Andreasen NC, Olsen S (1982): Negative versus positive schizophrenia: definition and validation. Arch Gen Psychiatry 39:789-794

Boccellari AA, Dilley JW (1992): Management and residential placement problems of patients with HIV-related cognitive impairment. Hosp Community Psychiatry 43:32-37

Breitbart W (1988): AIDS and Neuroleptic Malignant Syndrome. Lancet 24/31:1488-1489
Center for Disease Control and Prevention (1992): 1993 revised classification system for HIV infection and expanded surveillance case definition for AIDS among adolescents and adults. MMWR 41 (No. RR-17):1-19

Davis, JM, Baxter JT, Kane JM (1989): Antipsychotic Drugs. In Kaplan HI, Sadock BJ (eds), Comprehensive Textbook of Psychiatry/V Volume 2, 5th Ed. Baltimore: Williams and Wilkins, pp 1591-1626

Dixon WJ, Brown NB, Engleman L, Jennrich RI (1990): BMDP Statistical Software Manual, Berkeley: University of California Press

Fernandez F, Levy JK (1991): Psychopharmacotherapy of psychiatric syndromes in asymptomatic HIV infection and symptomatic HIV infection. Psychiatric Medicine 9:377394

Fernandez F, Levy J, Mansell PWA (1989): Management of delirium in terminally ill AIDS patients. Int J Psychi Med 19(2):165-172

Gilmer WS, Busch KA (1991): Neuropsychiatric aspects of AIDS and psychopharmacologic management. Psychiatric Medicine 9:313-329

Goodkin K (1988): Psychiatric disorders in HIV-spectrum illness. Texas Medicine 84:55-61

Harris MJ, Jeste DV, Gleghorn A, Sewell DD (1991): Newonset psychosis in HIV-infected patients. J Clin Psychiatry 52:369-376

Holmes VF (1989): Treatment of monosymptomatic hypochondriacal psychosis with pimozide in an AIDS patient. Am J Psychiatry 146:554-555

Hriso E, Kuhn T, Masdeu JC, Grundman M (1991): Extrapyramidal symptoms due to dopamine-blocking agents in patients with AIDS encephalopathy. Am J Psychiatry 148(11):1558-1561

Jeste DV, Wyatt RJ (1982): Understanding and Treating Tardive Dyskinesia, New York: Guilford Press, Inc.

Jeste DV, Lacro JP, Gilbert PL, Kline J, Kline N (1993): Treatment of late-life schizophrenia with neuroleptics. Schiz Bull 19:817-830

Kirk RE (1982): Split-plot factorial design: design with grouptreatment confounding. In Experimental Design: Procedures for the Behavioral Sciences, 2nd Ed. Monterey, CA: Brooks/Cole Publishing Co. pp 489-564

Masdeu JC, Yudd A, Van Heertum RL, Grundman M, Hriso E, O'Connell RA, Luck D, Camli U, King LN (1991): Single-photon emission computed tomography in human immunodeficiency virus encephalopathy: a preliminary report. J Nucl Med 32:1471-1475

Masliah E, Achim CL, Ge N, DeTeresa R, Terry RD, Wiley CA (1992): Spectrum of human immunodeficiency virusassociated neocortical damage. Ann Neurol 32:321-329

Miller D, Riccio M (1990): Non-organic psychiatric and psychosocial syndromes associated with HIV-1 infection and disease. AIDS 4:381-388

National Institute of Mental Health, 1976, AppendicesAbnormal Involuntary Movement Scale (AIMS). In Guy, W (ed) ECDEU Assessment Manual for Psychopharmacology, Revised, Rockville, MD: U.S. Department of Health, Education, and Welfare (Pub. No. (ADM) 76-338), pp 534-537

Ostrow D, Grant I, Atkinson H (1988): Assessment and 
management of the AIDS patient with neuropsychiatric disturbances. J Clin Psychiatry 49:14-22

Overall JE (1988): The brief psychiatric rating scale (BPRS): recent developments in ascertainment and scaling. Psychopharmacol Bull 24:97-99

Perry S, Jacobsen P (1986): Neuropsychiatric manifestations of AIDS-spectrum disorders. Hosp Community Psychiatry $37: 135-142$

Schluchter MD (1988): Analysis of incomplete multivariate data using linear models with structured covariance matrices. Stat Med 7:317-324
Schooler NR, Kane JM (1982): Research diagnoses for tardive dyskinesia. Arch Gen Psychiatry 39:486-487

Sewell DD, Jeste DV, Atkinson JH, Heaton RK, Hesselink JR, Wiley C, Thal L, Chandler JL, Grant I, HNRC Group (1994): HIV-associated Psychosis: A longitudinal study of 20 Cases. Am J Psychiatry 151:237-242

Simpson GM, Angus JW (1970): A rating scale for extrapyramidal side effects. Acta Psychiatr Scand 45(s212):11-12

Wragg RE, Jeste DV (1988): Neuroleptics and alternative treatments: Management of behavioral symptoms and psychosis in Alzheimer's disease and related conditions. Psychiatric Clinics of North America 11:195-214 